\title{
Análise da Revisão Cochrane: Antihistamínicos para a Constipação. Cochrane Database Syst Rev. 2015;11:CD009345.
}

\section{Analysis of the Cochrane Review: Antihistamines for the Common Cold. Cochrane Database Syst Rev. 2015;11:CD009345.}

\author{
Carmel STERRANTINO ${ }^{1}$, Gonçalo DUARTE²,3, João COSTA ${ }^{2,3}$, António VAZ-CARNEIRO $\square^{3,4}$ \\ Acta Med Port 2016 Mar;29(3):164-167 • http://dx.doi.org/10.20344/amp.7526
}

\section{RESUMO}

A constipação representa uma inflamação aguda e autolimitada da mucosa das vias aéreas superiores, que pode envolver uma ou todas de entre os seios perinasais, rinofaringe, orofaringe e laringe. É comum ter-se pelo menos um episódio por ano. Os sintomas da constipação, que podem incluir dor de garganta, crises esternutatórias, congestão nasal, rinorreia, cefaleias, mal-estar e febre baixa geralmente desaparecem dentro de alguns dias sem tratamento. $O$ agente etiológico da maioria das constipações é o rinovírus. Embora não esteja associado letalidade, a constipação é associada com morbidade significativa. Não existe vacina ou cura para a constipação e, portanto, o seu tratamento está centrado em aliviar os sintomas. Esta revisão Cochrane teve como objetivo sintetizar a evidência existente sobre o benefício clínico de anti-histamínicos, utilizados em monoterapia, em comparação com placebo ou nenhum tratamento, em crianças e em doentes adultos com constipação. Foram incluídos um total de 18 ensaios clínicos aleatorizados, com um total de 4342 participantes. Os principais resultados foram: 1) Anti-histamínicos têm um pequeno (dias um e dois de tratamento) efeito benéfico a curto-prazo sobre a gravidade dos sintomas globais em doentes adultos, embora este efeito não esteja presente no médio a longo prazo; 2) Os anti-histamínicos foram associados com um efeito benéfico não clinicamente relevante sobre os sintomas individuais (congestão nasal, rinorreia e esternutos); 3) Os anti-histamínicos não estão associados com um risco aumentado de efeitos adversos; 4) Nenhuma conclusão pode ser feita sobre a eficácia dos anti-histamínicos em populações pediátricas. A nossa interpretação dos resultados é que a evidência disponível é insuficiente para apoiar a prescrição ou a compra de anti-histamínicos de venda-livre para aliviar os sintomas da constipação sem componente alérgico.

Palavras-chave: Anti-Histamínicos/uso terapêutico; Constipação/tratamento; Revisão Sistemática.

\section{ABSTRACT}

The common cold is an acute, self-limiting inflammation of the mucosa of the upper airways, which may involve one or all the sinuses, nasopharynx, oropharynx and larynx. It is common to have at least one episode per year. Common cold symptoms, which may include sore throat, sneezing, nasal congestion, runny nose, headache, malaise and mild fever usually disappear within a few days without treatment. The causative agent of most colds is rhinovirus. Although not associated with mortality, common cold is associated with significant morbidity. There is no vaccine or cure for common cold and, therefore, their treatment is centered on relieving the symptoms. This Cochrane review aimed to synthesize the existing evidence about the clinical benefit of antihistamines, used as monotherapy, compared with placebo or no treatment in children and adult patients with common cold. A total of 18 randomized clinical trials with 4342 participants were included. Main results were: 1) Antihistamines have a small (days one and two) beneficial effect in the short term on the severity of overall symptoms in adult patients, although this effect is not present in the medium to long term; 2) antihistamines were not associated with a clinically significant beneficial effect on the individual symptoms (nasal congestion, rhinorrhea, and sneezing); 3) Antihistamines are not associated with an increased risk of adverse effects; 4) No conclusion can be made about the effectiveness of antihistamines in pediatric populations. Our interpretation of the results is that the available evidence is insufficient to support the prescription or buying OTC antihistamines to relieve the symptoms of common cold without allergic component.

Keywords: Common Cold/drug therapy; Histamine H1 Antagonists/therapeutic use; Systematic Review.

\section{QUESTÃO CLÍNICA}

Os anti-histamínicos são um tratamento eficaz nos sinais e sintomas da constipação?

\section{OBJETIVOS}

Avaliar a relação risco-benefício de anti-histamínicos, em monoterapia, na constipação em crianças e adultos.

Em particular, os autores tinham como objetivo avaliar se os anti-histamínicos aliviam sintomas globais e nasais (congestão nasal, rinorreia e esternutos); reduzem a duração do episódio; e são seguras (efeitos adversos).

\section{METODOLOGIA}

Revisão sistemática de ensaios clínicos aleatorizados comparando anti-histamínicos (quer sedativo ou não sedativo e administrado por via oral ou intranasal), em monoterapia, versus placebo ou nenhum tratamento, tanto em crianças (até 18 anos de idade) e adultos com constipação.

1. Department of Clinical and Experimental Medicine. Policlinico "G. Martino". University of Messina. Messina. Italy.

2. Laboratório de Farmacologia Clínica e Terapêutica. Faculdade de Medicina. Universidade de Lisboa. Portugal.

3. Centro de Estudos de Medicina Baseada na Evidência. Faculdade de Medicina. Universidade de Lisboa. Lisboa. Portugal.

4. Centro Colaborador Português da Rede Cochrane Iberoamericana. Lisboa. Portugal.

$\bowtie$ Autor correspondente: António Vaz Carneiro. avc@medicina.ulisboa.pt

Recebido: 15 de fevereiro de 2016 - Aceite: 16 de fevereiro de 2016 | Copyright @ Ordem dos Médicos 2016 
Os ensaios clínicos avaliando terapêuticas anti-histamínicas em combinação ou em doentes com um componente alérgico na sua doença foram excluídos. Os autores pesquisaram para estes ensaios clínicos aleatorizados nas seguintes bases de dados (agosto de 2015): Cochrane Central Register of Controlled Trials, que inclui o Cochrane Acute Respiratory Infections (ARI) Group's Specialised Register; MEDLINE; EMBASE; CINAHL; LILACS; e Biosis Previews. Os principais outcomes de interesse foram a mudança na gravidade dos sintomas globais (por exemplo, ausente, leve, moderada, grave) e alteração na duração dos sintomas globais. A extração de dados e a avaliação da qualidade dos ensaios clínicos incluídos foram feitas independentemente por dois dos autores.

\section{RESULTADOS}

Foram incluídos 18 ensaios clínicos aleatorizados, com um total de 4130 adultos e 212 crianças com um diagnóstico de constipação, tanto de ocorrência natural ou induzida experimentalmente. Todos os ensaios clínicos comparavam anti-histamínico em monoterapia versus placebo. A maioria dos estudos foram avaliados como sendo de boa qualidade, ou seja, com baixo risco de viés, embora alguns ensaios não tinham informação suficiente para um julgamento. Treze ensaios utilizaram um anti-histamínico sedativo como intervenção, o mais comum dos quais era maleato de clorfeniramina (cinco ensaios). Os ensaios clínicos mostraram diferenças relevantes em relação aos participantes, intervenções, resultados e desenho de estudos. Para além disso, muitos dos artigos publicados não disponibilizaram dados adequados. Assim, os autores realizaram apenas um número limitado de meta-análises. A maioria, destas incluem uma minoria dos ensaios clínicos disponíveis e fornecem uma avaliação incompleta da qualidade da evidência.

Nove ensaios clínicos, com 2906 participantes de idade adulta, avaliaram a alteração na gravidade dos sintomas globais. Quatro destes, utilizando anti-histamínicos sedativos, demostraram benefício. Os resultados de cinco desses nove ensaios que incluíram 1848 adultos foram analisados a fim de medir o efeito sobre os sintomas globais a curto (1 a 2 dias), intermediário (3 a 4 dias) e longo prazo (de 6 a 10 dias). No grupo que recebeu anti-histamínicos houve menos participantes sem efeitos benéficos (55\%) em comparação com o grupo que recebeu placebo (62\%) durante os primeiros dois dias (odds ratio [OR] $=0,74 ; 95 \%$ de intervalo de confiança [IC] 0,60 a 0,92; 3 ensaios). Não se observou efeito a médio e longo prazo. Em relação à alteração da gravidade dos sintomas individuais, houve uma diminuição não clinicamente significativa em rinorreia dos dias 2 - 4 e em espirros em todos os dias de tratamento nos participantes tratados com anti-histamínicos sedativos.

O perfil de segurança foi avaliado numa análise com 2 590 participantes. Duzentos e cinquenta nove participantes de $1284(20,2 \%)$ e 230 de 1306 (17,6\%) apresentaram pelo menos um efeito adverso no grupo anti-histamínicos e placebo, respetivamente. Este aumento da proporção de participantes com eventos adversos como sedação, não foi estatisticamente significativa (OR 1,18; IC de 95\% 0,97 $1,44)$. Os resultados dos dois ensaios com participantes em idades pediátricas foram considerados pelos autores como tendo resultados contraditórios.

A Tabela 1 mostra o summary of findings para as principais comparações para anti-histamínicos versus placebo em doentes constipados.

\section{CONCLUSÕES}

Anti-histamínicos em monoterapia parecem ter um efeito benéfico limitado e de curta duração sobre os sintomas globais da constipação. Uma meta-análise analisando anti-histamínicos sedativos versus placebo mostrou um efeito em relação a sintomas individuais, tais como congestão nasal, rinorreia e espirros, sem que esta diferença fosse clinicamente relevante. Isto pode potencialmente ser explicado pela inibição da estimulação colinérgica, predominantemente presente como efeito dos anti-histamínicos sedativos quando comparados com os anti-histamínicos não sedativos. Finalmente, não há evidência de que os anti-histamínicos estejam associados com um risco acrescido de efeitos adversos. É provável que alguns efeitos adversos reportados possam, na verdade, ser atribuídos a sintomas da doença e não do medicamento.

\section{COMENTÁRIO}

Os anti-histamínicos ou antagonistas dos recetores $\mathrm{H} 1$ possuem a capacidade de inibir várias ações histamínicas. Eles atuam de modo a evitar a interação com os recetores de histamina através da competição com a histamina, em vez de inibir a libertação de histamina e são úteis na prevenção ações histamínicas, tais como a rinite alérgica. ${ }^{2}$ Esta classe de fármacos está dividida em dois grupos: sedativo e não sedativo.

Os anti-histamínicos sedativos, também chamados anti-histamínicos de primeira geração, estão associados a vários eventos adversos, em grande parte devido à sua propensão para atravessar a barreira hematoencefálica e à sua atividade colinérgica, causando sintomas de sonolência e concentração reduzida, bem como boca seca, visão baça e retenção urinária. . $^{3,4}$ Os anti-histamínicos de segunda geração são lipofóbicos e atravessam a barreira hematoencefálica em muito menor medida, evitando, portanto, o envolvimento do sistema nervoso central e efeitos colinérgicos. ${ }^{3}$ Uma revisão recente ${ }^{5}$ relatado alguns efeitos positivos de combinações anti-histamínicos com analgésico-descongestionantes para o alívio sintomático da constipação. No entanto, também foram reportados numerosos efeitos adversos.

Nesta revisão a única evidência de um efeito benéfico limitado sobre os sintomas individuais foi encontrado na análise de agentes de primeira geração. Nas análises da gravidade dos sintomas globais foram incluídos apenas um pequeno número de ensaios. O benefício de curto prazo resultou de uma análise combinada de três estudos (1490 participantes), e foi de uma pequena magnitude do efeito 


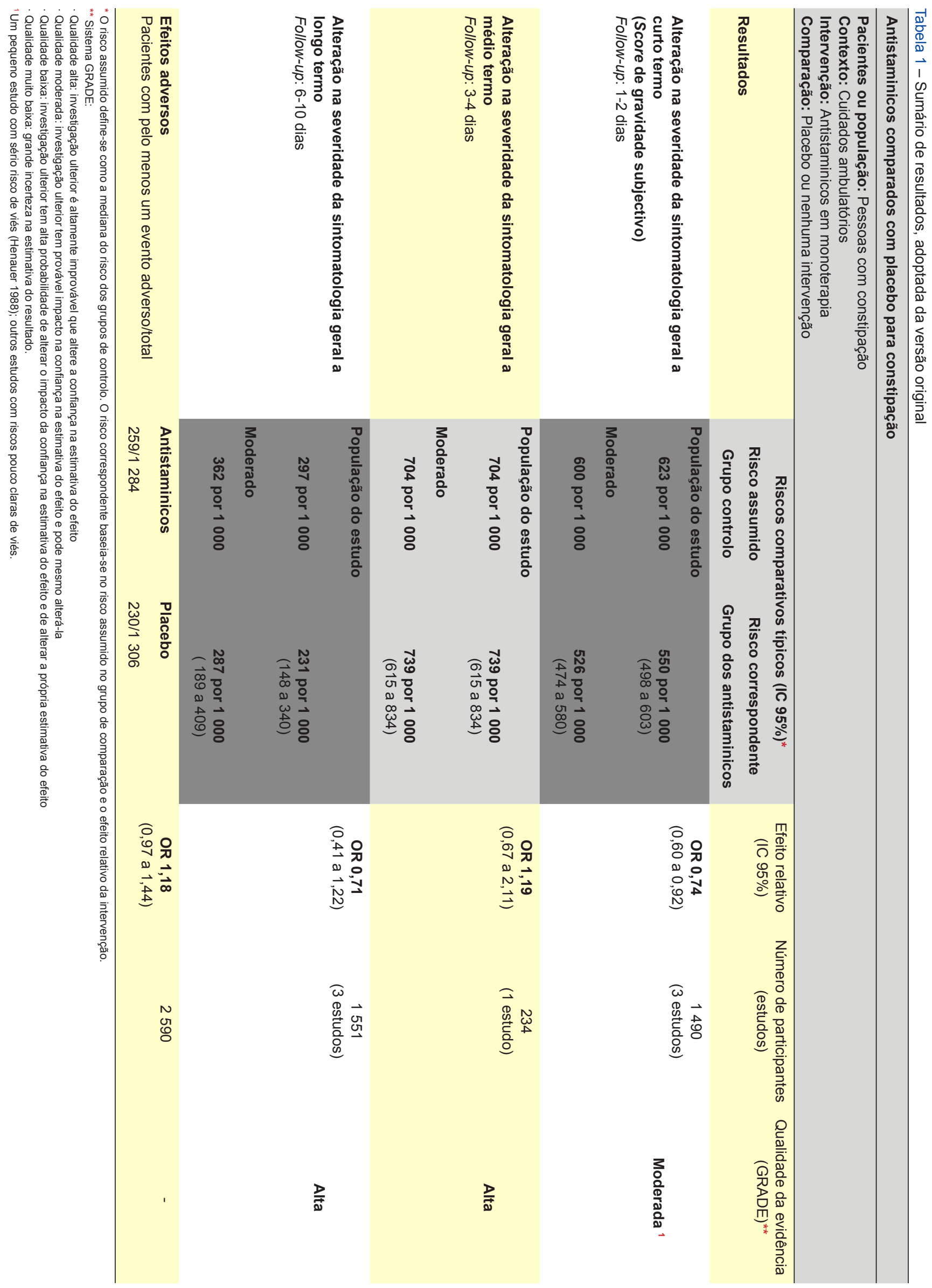


(7\% menos doentes no grupo anti-histamínico deixar de ter efeitos benéficos). Em dois dos ensaios (com 1427 participantes) os fármacos utilizados foram clorfeniramina e tonzilamina, ambos anti-histamínicos sedativos. Embora a inibição da estimulação colinérgica pudesse apoiar esta conclusão, a magnitude do efeito sobre os sintomas individuais foi muito pequena, com a maior diferença média entre os dois grupos sendo de 0,24 - 0,35, com uma mudança de um ponto significando uma alteração de uma categoria de gravidade (por exemplo, severa a moderada). Com base nesta análise, não existe um efeito clinicamente significativo na obstrução nasal, rinorreia ou espirros e o efeito benéfico sobre a gravidade dos sintomas globais está limitada aos dois primeiros dias de tratamento.

\section{REFERÊNCIAS}

1. Heikkinen T, Jarvinen A. The common cold. Lancet. 2003;361:51-9.

2. Mann KV, Crowe JP, Tietze KJ. Non-sedating histamine H1-receptor antagonists. Clin Pharm. 1989;8:331-44.

3. Gonzalez MA, Estes KS. Pharmacokinetic overview of oral second-generation H1 antihistamines. Int J Clin Pharmacol Ther. 1998;36:292-300.
Em conclusão, esta evidência fragmentária não fornece uma base sólida sobre a qual possam ser emitidas conclusões. O mesmo se aplica em crianças.

\section{IMPLICAÇÕES PARA A PRÁTICA CLÍNICA}

1. Os anti-histamínicos têm um pequeno efeito benéfico a curto-prazo (um ou dois dias de tratamento) sobre a gravidade dos sintomas globais da constipação em doentes adultos;

2. A administração de anti-histamínicos em doentes com constipação parece ser segura;

3. A evidência disponível é insuficiente para apoiar a prescrição ou a compra de anti-histamínicos de venda-livre para aliviar os sintomas da constipação sem um componente alérgico.
4. Rossi S. Australian Medicines Handbook. $11^{\text {th }}$ edition. Adelaide: Australian Medicines Handbook Pty Ltd; 2010.

5. De Sutter AI, van Driel ML, Kumar AA, Lesslar O, Skrt A. Oral antihistamine-decongestant-analgesic combinations for the common cold. Cochrane Database Syst Rev. 2012;2.

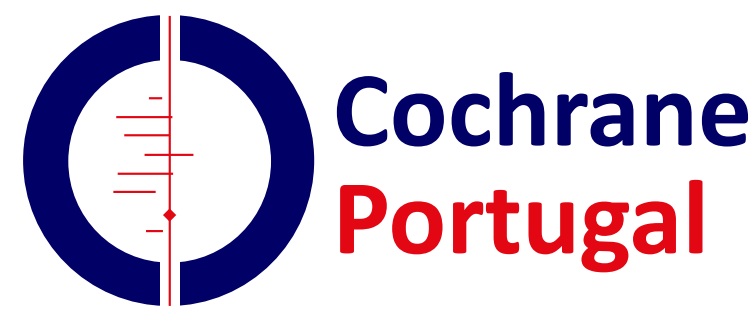

